\title{
Neovascularization Assessment in Canine Mammary Carcinoma - A Case Report
}

\author{
Alexandra IRIMIE ${ }^{1 *}$, Alexandru Raul POP ${ }^{2}$, Vlad Alexandru ILAȘ², Robert PURDOIU ${ }^{3}$, Cornel CĂTOI ${ }^{1}$ \\ ${ }^{1}$ Pathology Department, University of Agricultural Sciences and Veterinary Medicine, 400372, Cluj- \\ Napoca, Romania \\ ${ }^{2}$ Clinical Reproduction Department, University of Agricultural Sciences and Veterinary Medicine, \\ 400372, Cluj-Napoca, Romania \\ ${ }^{3}$ Radiology and Imagistic Laboratory, University of Agricultural Sciences and Veterinary Medicine, \\ 400372, Cluj-Napoca, Romania \\ *corresponding author: alexandra.irimie2010@gmail.com
}

Bulletin UASVM Veterinary Medicine 73(2) / 2016,

Print ISSN 1843-5270; Electronic ISSN 1843-5378

DOI:10.15835/buasvmcn-vm: 12087

\begin{abstract}
The frequency of mammary gland tumors in canines is three times higher than in women. Contrast enhanced ultrasonography (CEUS) is a noninvasive clinical method, that uses special contrast agents (CAs), their role being to layout the microvasculature of different lesions. The aim of this particular method, in this case, was to establish if we can evaluate the malignancy of a mass, given the fact that neovascularization is a malignancy marker. The case is represented by a Silky Terrier breed female dog, 5 years old, that was presented initially with an enlarged polycystic mammary gland and a nervous lactation. The female was initially diagnosed with polycystic mastosis. After 2 more months the mass became denser and enlarged. Before the ovariohysterectomy and unilateral mastectomy surgeries have taken place, a B-Mode standard ultrasound, CEUS and a pulmonary X-ray were performed. Our results integrate this case in "fast in" and "slow in" (type 2 curves). The sample was fixed immediately after excision in $10 \%$ neutral buffered formalin for 24 hours and embedded in paraffin wax. Serial sections were cut at $4 \mu \mathrm{m}$ from each block and stained with hematoxylin and eosin (H\&E) and additional sections mounted on poly-L lysine coated slides. The immunohistochemistry process was realized by using rabbit anti-VEGF receptor 2 antibodies. The histological diagnosis was established as a simple cystic papillary carcinoma with a malignancy grade 2 . The mean value of the microvessel density (MVD) was 13.75 which are a low MVD. We cannot determine a correlation between CEUS and a tumor's malignancy, and so further studies are needed.
\end{abstract}

Keywords: CEUS, malignancy, microvessel density, neovascularization

\section{INTRODUCTION}

Contrast enhanced ultrasonography (CEUS) is an ultrasound method that uses different contrast agents (CAs) and this type of ultrasound technique was established in order to overcome the standard B-Mode ultrasonography. The role of these CAs is to layout the microvasculature of different lesions and tissues. The use of this type of ultrasound allows the observation of microvessels in real time.
The main treatment option in a mammary tumor is the surgical one and given the fact that an owner is reluctant to this, we tried to establish if a mass can be evaluated as a malignant tumor due to the fact that neovascularization can be a prognostic marker (Gal et al., 2011).

The Update of the Guidelines of the European Federation of Societies for Ultrasound in Medicine and Biology (EFSUMB), published in 2012, on non- 
hepatic lesions, stated that a pattern indicating malignant criteria using CEUS cannot be identified. Thus using contrast enhanced ultrasonography examination can be a topic for further research (Piscaglia et al. 2012).

Even thou many studies can be found on human patients with breast lesions, using CEUS to determine the malignancy in mammary gland tumors in dogs were not found. Studies using CEUS on canine patients with different pathologies such as pancreas masses or pancreatitis, renal perfusion, prostate masses or different hepatic disorders, are many but on mammary tumors are not.

The aim of this study was to perfect the contrast enhanced ultrasonography method on a canine patient and apply it to mammary tumors, by determining the degree of vascularization, fact that can be confirmed through microvessel density (MVD) quantification. As stated earlier, neovascularization can be prognostic factor. $\mathrm{Du}$ (2008) found a significant correlation between MVD and CEUS parameters.

\section{MATERIALS AND METHODS}

The study was conducted on a Silky Terrier breed female dog, 5 years old, that was initially presented at the Reproduction Clinic, Faculty of Veterinary Medicine Cluj-Napoca, and 2 months after the estrus cycle was ended, with an enlarged polycystic mammary gland and a nervous lactation. The female was diagnosed, initially by ultrasound exam, with polycystic mastosis and treatment and diet was established. The nervous lactation was successfully treated but after 2 more months the mass became enlarged and denser in consistency. The owner did not want to reproduce her again (she had previously one litter) and decided to spay her and have the mass removed, but firstly to do a cytological exam. We recommended a unilateral mastectomy, because of the mass's possible malignancy. Before the surgery, we did the ultrasound examination using a standard B-Mode, as well as a pulmonary X-Ray. After the region of interest was established we conducted de contrast enhanced ultrasound. After the X-Ray was negative for metastasis and after ultrasound examinations, the ovariohysterectomy and unilateral mastectomy were performed.

\section{Ultrasound (US) in B-Mode and CEUS}

The ultrasound examination (US) was performed, at the Internal Medicine Clinic in our Faculty, using an Esaote MyLab ${ }^{\text {TM }} 40$ VET system and a linear transducer with a 7.5 MHz frequency. B-Mode and ultrasound exam was performed to determine a region of interest (ROI). The patient was not sedated. It was administered intravenously in bolus $1 \mathrm{ml} / 10 \mathrm{~kg}$ of prepared contrast agent (CA) SonoVue $(8 \mu \mathrm{l} / \mathrm{ml}$ sulphur hexafluoride microbubbles, Bracco, Italy). A 3-minute video was obtained and processed with ImageJ software using specialized relative intensity units (riu).

\section{CEUS parameter analysis}

The changes in intensity were analyzed as time-intensity curves, the intensity being measured in relative intensity units. 3 regions of interest (ROIs) were selected in the most enhanced area within the tumor and only the mean CEUS parameter value was regarded. Each ROI was measured at 800 relative intensity units. The curves were analyzed for these indices: baseline intensity (BI; echogenic value of the noncontrast tissue; riu), arrival time (AT; the first point of the curve above the baseline intensity followed by a rise - arrival moment the contrast agent reaches the tissue; seconds), peak intensity (PI; the max. value of the contrast agent; riu), rise time (RT; from AT to PI - the time the contrast agent needs to reach a maximum value; seconds), enhancement intensity (EI; the time the contrast agent remains in the tissue at high value - approx. $80 \%$ out of the PI; seconds), wash in (the point where it can be observed a constant rise until EI; units/seconds) and wash out (from EI a constant decrease of contrast; units/seconds).

\section{Cytology}

The cytological examination was performed using a fine needle aspiration and a Diff-Quick staining.

\section{Histopathology}

The sample was retrieved after ovariohysterectomy and a unilateral mastectomy with the additional regional lymph-node. The sample was fixed immediately after excision in $10 \%$ neutral buffered formalin for 24 hours and embedded in paraffin wax. Serial sections were cut at $4 \mu \mathrm{m}$ from each block and stained with hematoxylin and eosin (H\&E) respecting the ultrasound section (in this case, the longitudinally trough the tumor). 


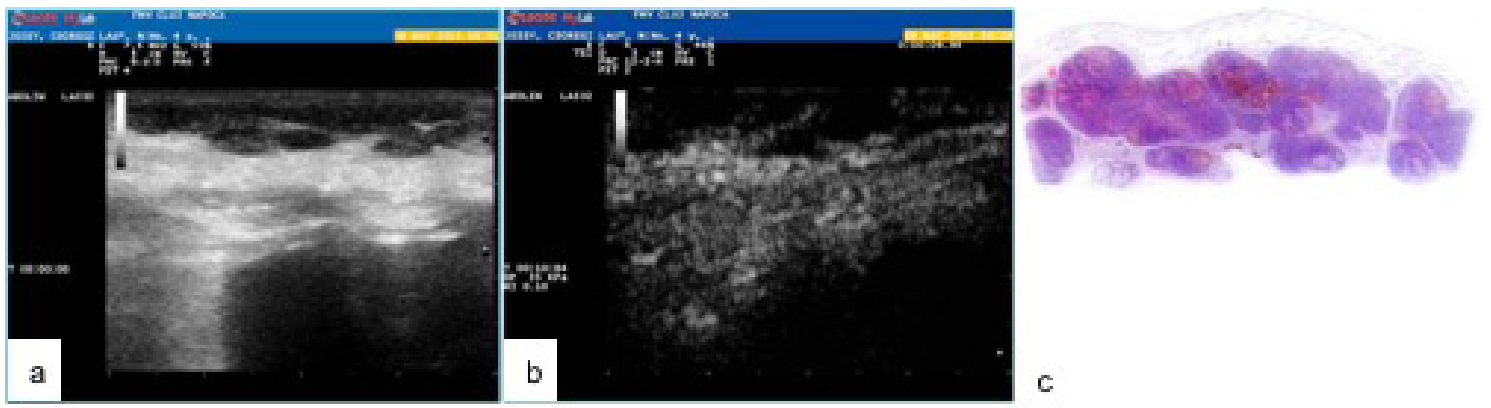

Fig. 1. (a) with a B-Mode ultrasound we select the ROI and evaluate the aspect of the mass; (b) CEUS - the vasculature can be seen in the intratumoral stroma; (c) a small magnification of the histological slide.

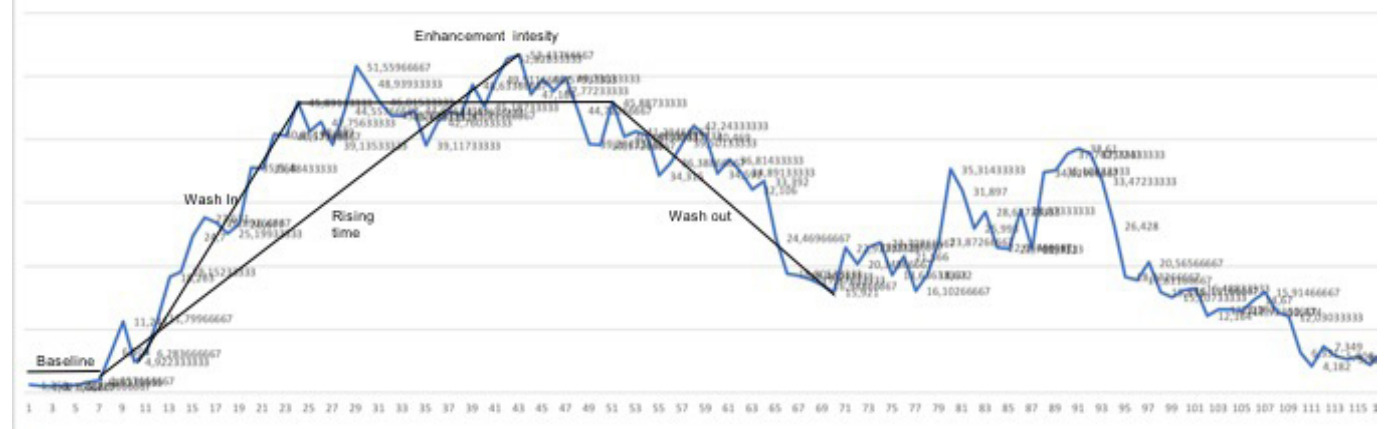

Fig. 2. Time-intensity curve with CEUS parameters.

\section{Immunohistochemistry}

After the embedment in paraffin wax, sections were cut from the block and mounted on poly-L lysine coated slides. The process was realized by using rabbit anti-VEGF receptor 2 antibodies (dilution 1:100, Linaris Biologische Produkte $\mathrm{GmbH}$, Germany). The immunohistochemical protocol was performed using the Leica Bond Max automatic system. We calculated MVD by counting the microvessels in 5 fields at 200X magnification. The mean value was then calculated.

The cytological, histopathological and immunohistochemical examinations were performed at the Pathology Department in our Faculty and reviewed by 2 separate examinators.

\section{RESULTS AND DISCUSSION}

The ultrasound images show multiple hypoecogenic and avascular cysts with a fine medium echogenic line between these cysts, a line that represents the neoplasic mass stroma (fig. 1a). After the region of interest (ROI) was set, CEUS was the performed. The results after CEUS were that a layout of the intratumoral microvessels can be observed in the region of the tumoral stroma, fact that can be demonstrated trough immunohistochemistry in figures 3 (b), (c), and (d). After video analysis, the contrast pattern of the lesion was determined: the enhancement order was centripetal, the distribution of contrast agent was heterogeneous, perfusion defects were absent and the margin type was well defined. The enhancement degree relative to the normal breast tissue at peak intensity (PI) was iso-enhanced. Our CEUS parameters are reported in Table 1.

The author Du (2008), classified the intensity curves into 4 types according to the median value they obtained (11.8 intensity value) of the washin and the wash-out slope of curve. They defined "fast in" as the wash in slope is greater than the median value, "slow in" as wash in is the median value or less, "fast out" as wash out is the median value or less, and "slow out" as wash out is greater than the median value.

Our results, compared to the classification Du (2008) proposed, integrate this case in "slow in" and "fast out" (type 2). In this type 2 of curve Du (2008) observed that only 6 out of 53 breast lesions were included. Type 2 curve basically means that the contrast agent (CA) reached the tumor lesion quite slow, but the wash out was 
Tab. 1. The values of CEUS parameters

\begin{tabular}{ccc}
\hline CEUS parameters & & Value \\
\hline BI & Relative intensity units (riu) & 1.97 \\
\hline PI & riu & 53.43 \\
AT & seconds & 7.14 \\
TTP & riu & 40.74 \\
\hline RT & seconds & 34.94 \\
\hline EI & seconds & 27 \\
\hline Wash-in & units/seconds & 2,93 \\
\hline Wash-out & units/seconds & $-1,58$ \\
\hline
\end{tabular}

fast. This can be interpreted by a lower number of vessels. Du (2008) observed that the wash in and peak intensity were higher in malignant tumors, fact that suggest a stronger enhancement and a higher perfusion flow. If the area under the curve in larger, it can be explained by high degree of vascularization and by the retained contrast agent in the many new vessels.

After the cytological examination, no tumor cells were observed and the results were inconclusive. The histological report stated: Malignant mass, represented by the proliferations with a tubular-papillary aspect and a large area of cystic papillary carcinoma. Intratumoral ductal structures show the multilayered epithelium, highlighting in some places myoepithelial cells near the neo-formed ducts. The tumor cells show large nuclei, vesicular macronucleoli (1 or 2 ; nuclear grade 2), the number of mitosis being $12 / 10$ fields. In the areas with the cystic papillary aspect, papillary structures are observed, often large, with a supportive stroma; in the cystic cavity, an acidophilic secretion and cell debris can be observed (Fig. 3 a). In the tumor mass numerous small areas of necrosis and hemorrhage are observed. The capsule is thin; the wound edges not being infiltrated by tumor cells. The histological diagnosis was established as a simple cystic papillary carcinoma with a malignancy grade 2 (fig. 3). The malignancy grade was established using Peña's criteria (Goldschimdt et al., 2011).

Trough immunohistochemistry, we evaluated the microvessel density (MVD), but only the new small vessels and only in the intratumoral areas (without the tumor capsula). The mean value of the MVD was 13.75 which is a low MVD compared to Graham (1999) obtained in his study. In his study, the MVD for malignant lesions was 32 and no difference in MVD was found between the benign and malignant tumors without metastasis.

Weidner (1995), an author who intensively studied angiogenesis, stated in his work, that an association between intratumor microvessel density and tumor aggressiveness can be explained in several ways: a primary tumor with a high angiogenesis and intratumor microvessel density is more likely to produce distant sites; solid tumors are formed by two components (tumor cells and the stroma the cells induce) and the intratumor microvessel density could be a successful measure, that a tumor has in forming the important stroma; the density of the small vessels bed within a tumor is likely a direct correlation with the time and space that a tumor cell needs to pass and determine metastasis. If the space is wider, the number of circulating cells and the possibility of metastasis development is greater. Of course, angiogenesis alone is not sufficient in the development of metastasis (the cell proliferation needs to take place and they need to penetrate the tissue and vessels and survive the migration until the new site).

In a study, the author obtained a significant correlation between tumor malignancy grade and microvessel density, fact that clearly shows the use as an aggressiveness marker in canine mammary carcinomas (Gal et al., 2011). Given this fact, we can state that the tumor we diagnosed has a low aggressive behavior after MVD quantification, even thou the malignancy grade was moderate. Fact that can only be presumed after CEUS. The prolonged time of the contrast agent wash in, indicates a low degree of vascularization. If the tumor was very well vascularized and the MVD was high, the CEUS curve should have had "fast in" and "fast out" (a type 1 curve) characteristics (Du 


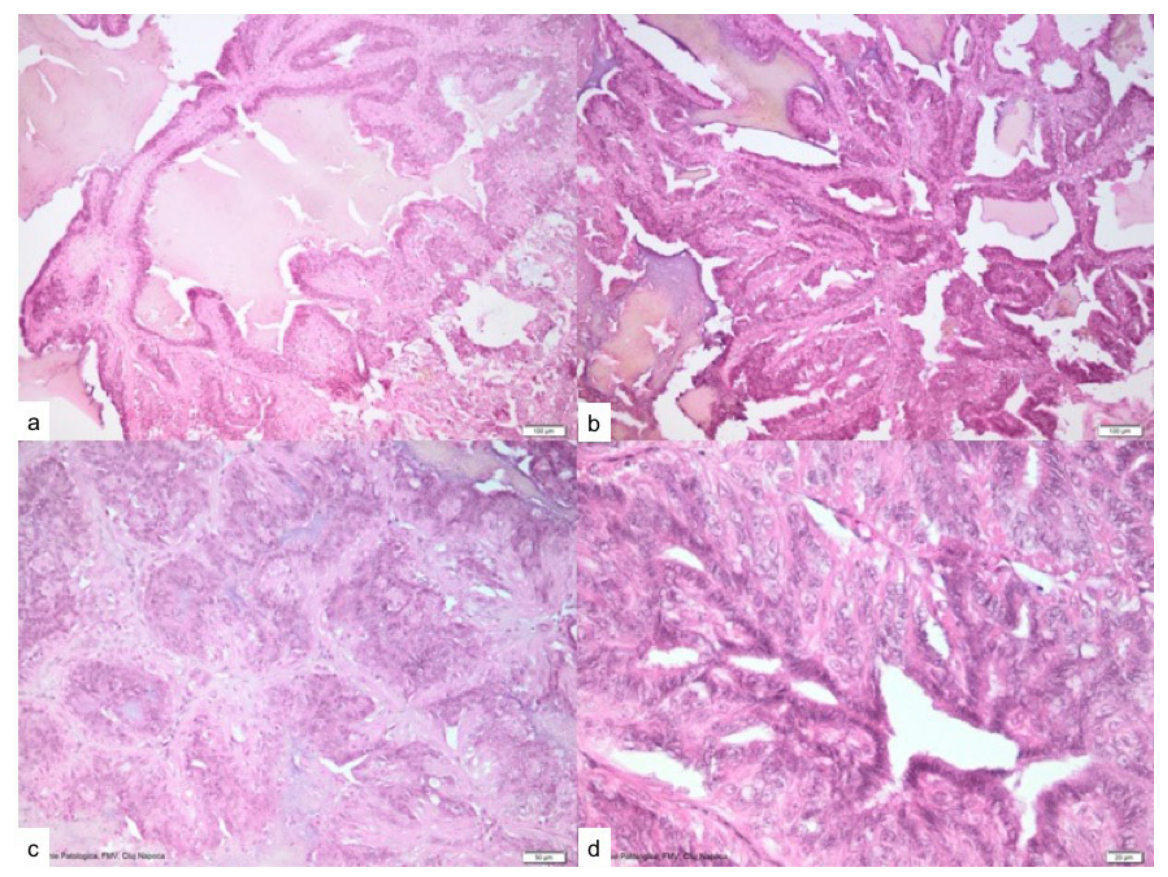

Fig. 3. Simple Cystic papillary carcinoma, grade 2. a. b. 100X; c. 200X; d. 400X; H\&E stain
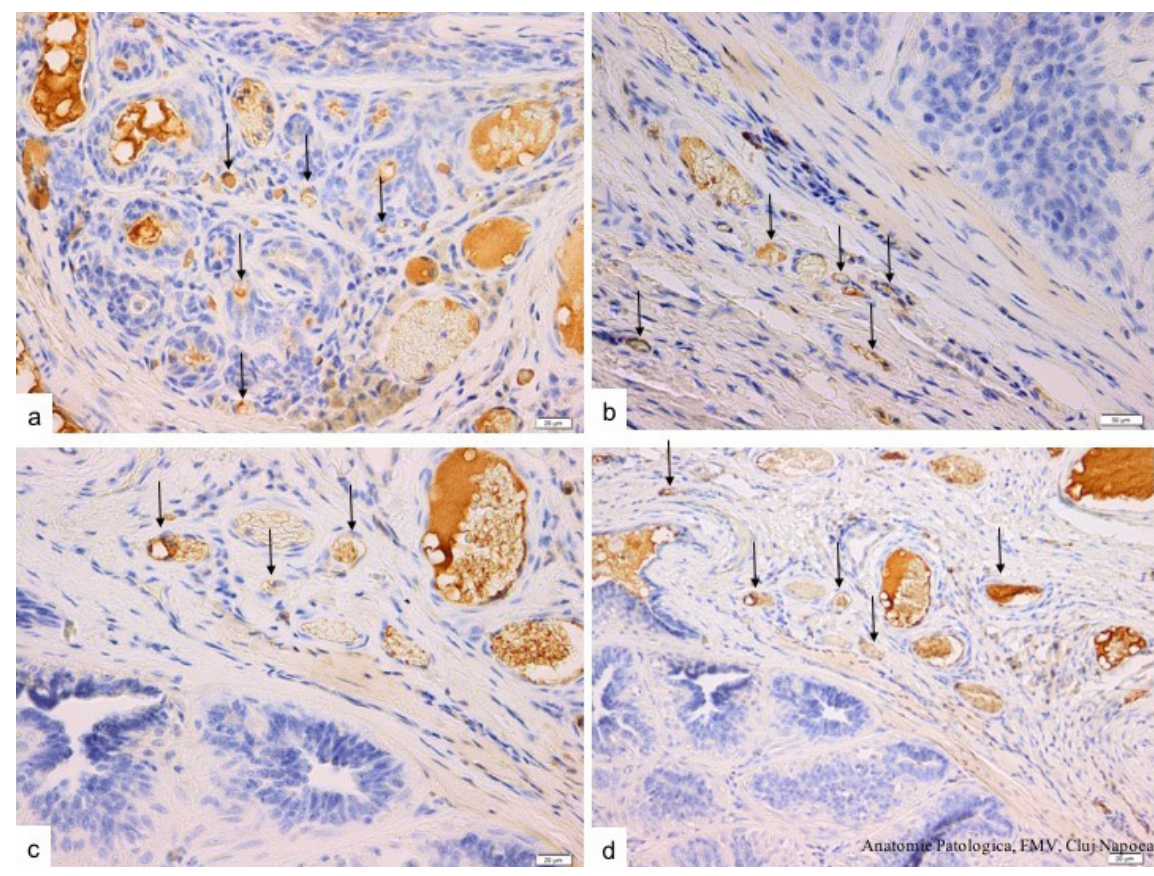

Fig. 4. Immunohistochemical staining with antibody anti-VEGF receptor 2 . Positive capillary endothelial cells marked brown (black arrows); (a) intratumoral microvessels - note that only the small vessels were marked with arrows (1 to 4 endothelial cells); (b), (c), (d) positive marked microvessels in the intratumoral stroma; a. d. 200X; b. c. $400 X$.

et al, 2008). In the same study, the author stated that no significant differences between malignant and benign groups were found, but statistical associations between CEUS parameters and MVD were demonstrated. Du (2008) stated that these parameters associate more with the degree of vascularization than a malignancy grade. This statement applies to our case study as well. 
The advantages of this method compared $\mathrm{cu}$ contrast computed tomography (CT), for example, are that is a non-invasive procedure, the patient does not have to be under general anesthesia and the contrast agent as well as CEUS are very safe to use on small animals (Seiler et al., 2013).

In human cancer patients, with different sites of disease such as colorectal, breast, lung and ovarian tissues, the expression VEGF is enhanced and is correlated with a high neovascularization (Zheng et al., 2003).

Du ( 2008), observed in his study on 53 breast lesions in women that CEUS parameters of breast lesions are more closely correlated with MVD than VEGF expression and are not correlated with the malignancy or benignity of breast tumors.

\section{CONCLUSIONS}

Given the fact that the type 2 curve was seen in only 6 out of 53 breast lesion in Du (2008) study, we could not draw a clear conclusion if the tumor had a low or a high degree of vascularization after CEUS. The wash in or slope 1 indicates a low vascularization, but the wash out and the area under curve indicate a high number of vessels.

We found a low microvessel density after immunohistochemistry, fact that can indicate a low aggressive tumor behavior. But the malignancy grade established after histopathology indicated that the tumor was moderate or intermediate (grade 2).

The results we obtained after CEUS are inconclusive and a higher number of patients are needed in order to correlate CEUS parameters with the malignancy grade, MVD and VEGF expression.
We cannot determine a correlation between CEUS and the tumor's malignancy.

\section{REFERENCES}

1. Du J, Li FH, Fang H, Xia JG, Zhu CX (2008). Correlation of Real-time Gray Scale Contrast-Enhanced Ultrasonography with Microvessel Density and Vascular Endothelial Growth Factor Expression for Assessment of Angiogenesis in Breast Lesions. J Ultrasound Med 27:821-831.

2. Gal AF, Baba AI, Miclaus V, Titilincu A, Cuc C, Taulescu $M$, Catoi C (2011). Aspects regarding angiogenesis and vasculogenic mimicry in canine mammary tumors. Revista Romana de Medinica de Laborator 19(3/4): 289 - 302.

3. Goldschmidt M, Peña L, Rasotto R and Zappulli V (2011). Classification and Grading of Canine Mammary Tumors. Vet Pathol 48: 117.

4. Graham JC and Myers RK (1999). The Prognostic Significance of Angiogenesis in Canine Mammary Tumors. J Vet Intern Med 13:416-418.

5. Piscaglia F et al. (2012). The EFSUMB Guidelines and Recommendations on the Clinical Practice of Contrast Enhanced Ultrasound (CEUS): Update 2011 on nonhepatic applications. Ultraschall in Med 33: 33 - 59.

6. Seiler GS, Brown JC, Reetz JA, Taeymans O, Bucknoff B, Rossi F, Ohlerth S, Alder D, Rademacher N, Drost WT, Pollard RE, Travetti O, Pey P, Saunders JH, Shanaman MM, Oliveira CR, O’Brien RT, Gaschen L (2013). Safety of contrast-enhanced ultrasonography in dogs and cats: 488 cases (2002-2011). J Amer Vet Med Assoc 242(9): 12551259.

7. Weidner N (1995). Intratumor Microvessel Density as Prognostic Factor in Cancer. American Journal of Pathology 147: 9-19.

8. Zheng S, Han MY, Xiao ZX, Peng JP, Dong Q (2003). Clinical significance of vascular endothelial growth factor expression and neovascularization in colorectal carcinoma. World J Gastroenterol 9: 1227-1230. 